\title{
Spectral Modeling with APEC
}

\author{
Nancy S. Brickhouse and Randall K. Smith \\ Harvard-Smithsonian CfA, 60 Garden St., Cambridge, MA 02138
}

\begin{abstract}
.
The Astrophysical Plasma Emission Code (APEC) collaboration now provides public models for X-ray spectra of collisional equilibrium plasmas. These models facilitate the diagnosis of temperature, density, elemental abundance, charge state, and optical depth. We report benchmarking studies of the APEC models from the Emission Line Project, a project to test these models using high quality stellar coronal spectra. We discuss the implications of the benchmarked atomic data for non-equilibrium collisional models as well. Finally, we discuss the extension of APEC to other applications, such as opacity models for AGN.
\end{abstract}

\section{Introduction}

APEC V1.3.1 (http://cxc.harvard.edu/atomdb) provides detailed spectral analysis capability for X-ray grating data. APEC line and continuum models are reasonably accurate and complete for collisional ionization equilibrium for $\lambda<$ $40 \AA$. Ongoing project efforts include non-equilibrium ionization modeling and opacities for X-ray photoionized plasmas. We note several issues that require better atomic data.

\section{Collisionally Ionized Plasmas}

Our goal is to provide calculations of populations and corresponding line emissivities for all levels $\geq n=5$ for $\mathrm{K}$ - and L-shell ions and $n=3$ for M-shell ions for the Chandra and XMM-Newton gratings. When available, accurate wavelengths from laboratory measurements replace the original theoretical wavelengths. Good spectral coverage and accurate wavelengths allow diagnostic applications even in heavily blended spectral regions (e.g. near Ne IX, Ness et al. 2003). We will soon complete the line database in the range $40 \AA<\lambda<100 \AA$.

Published uncertainty estimates suggest that $30 \%$ accuracy in diagnostic ratios is a reasonable goal for strong lines. We note significant problems with other groups' calculations of $\mathrm{H}$ - and He-like line emissivities, stemming from incorrect energy extrapolations and inadequate treatment of recombination-driven cascades, (Smith et al. 2001), and our calculations should now meet this goal. The Emission Line Project is providing observational tests (Brickhouse \& Drake 2000). While issues with Fe XVII are well known (Silver et al.; Beiersdorfer et al., these proceedings), similar discrepancies exist for other Fe L-shell ions as well (Fig. 1). We are now adding inner-shell transitions that become important for ionizing and recombining plasmas. Fig. 1 shows a wealth of satellite lines from 

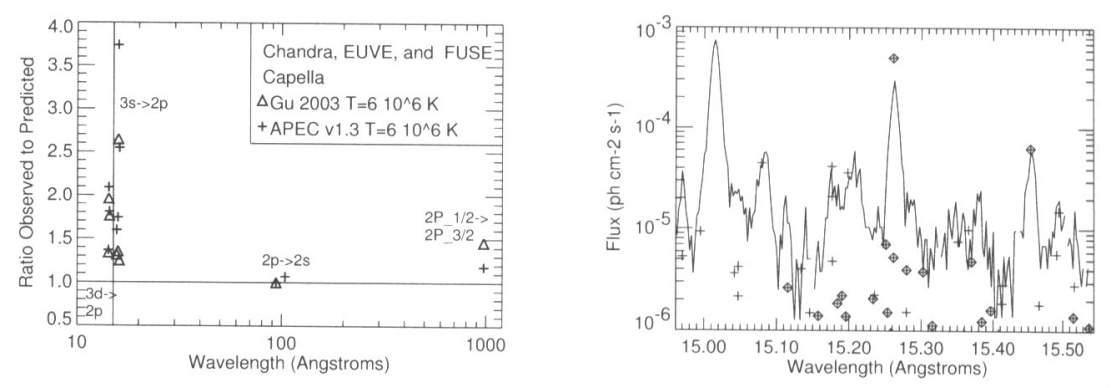

Figure 1. Left. Observed vs predicted line fluxes for Fe XVIII in Capella (P. Desai et al. 2003, in prep.). While the Gu (2003) calculations somewhat improve the agreement among the X-ray lines, a $40 \%$ discrepancy remains between the EUV and X-ray resonance lines. Right. Chandra Capella spectrum with APEC lines marked (+). Lines not due to Fe XVII DR are overplotted with $\diamond$ symbols.

Fe XVII that could help diagnose the ionization state; however, line-resolved calculations and laboratory wavelengths exist for only a few satellites.

\section{Opacity Models}

Krongold et al. (2003) have recently applied the APEC line list, plus additional inner-shell transitions, to the $900 \mathrm{ks}$ Chandra summed spectrum of NGC 3783, using only a few parameters to fit more than 100 absorption features. A discrepancy exists between the best-fits to the Fe M-shell unresolved transition array (UTA) and to the $\mathrm{Si}$ absorption lines. To resolve this discrepancy, complete and unabbreviated models are needed.

Acknowledgments. The authors acknowledge APEC collaborators Duane Liedahl and John Raymond. Support for this project is provided by NASA NAS8-39083 to SAO for the CXC and LTSA NAG5-3559.

\section{References}

Brickhouse, N. S., \& Drake, J. J. 2000, Rev. Mex. de Astronomia y Astrofisica Conf. Ser., 9, 24

Gu, M.-F. 2003, ApJ, 582, 1241

Krongold, Y., Nicastro, F., Brickhouse, N. S., Elvis, M., Liedahl, D. A., \& Mathur, S. 2003, ApJ, 597, 832

Ness, J.-U., Brickhouse, N. S., Drake, J. J., \& Huenemoerder, D. P. 2003, ApJ, 598, 1277

Smith, R. K., Brickhouse, N. S., Liedahl, D. A., \& Raymond, J. C. 2001, ApJ, 556, L91 